Document downloaded from:

http://hdl.handle.net/10251/105475

This paper must be cited as:

Carreño, A.; Vidal-Ferràndiz, A.; Ginestar Peiro, D.; Verdú Martín, GJ. (2017). Spatial modes for the neutron diffusion equation and their computation. Annals of Nuclear Energy. 110:1010-1022. doi:10.1016/j.anucene.2017.08.018

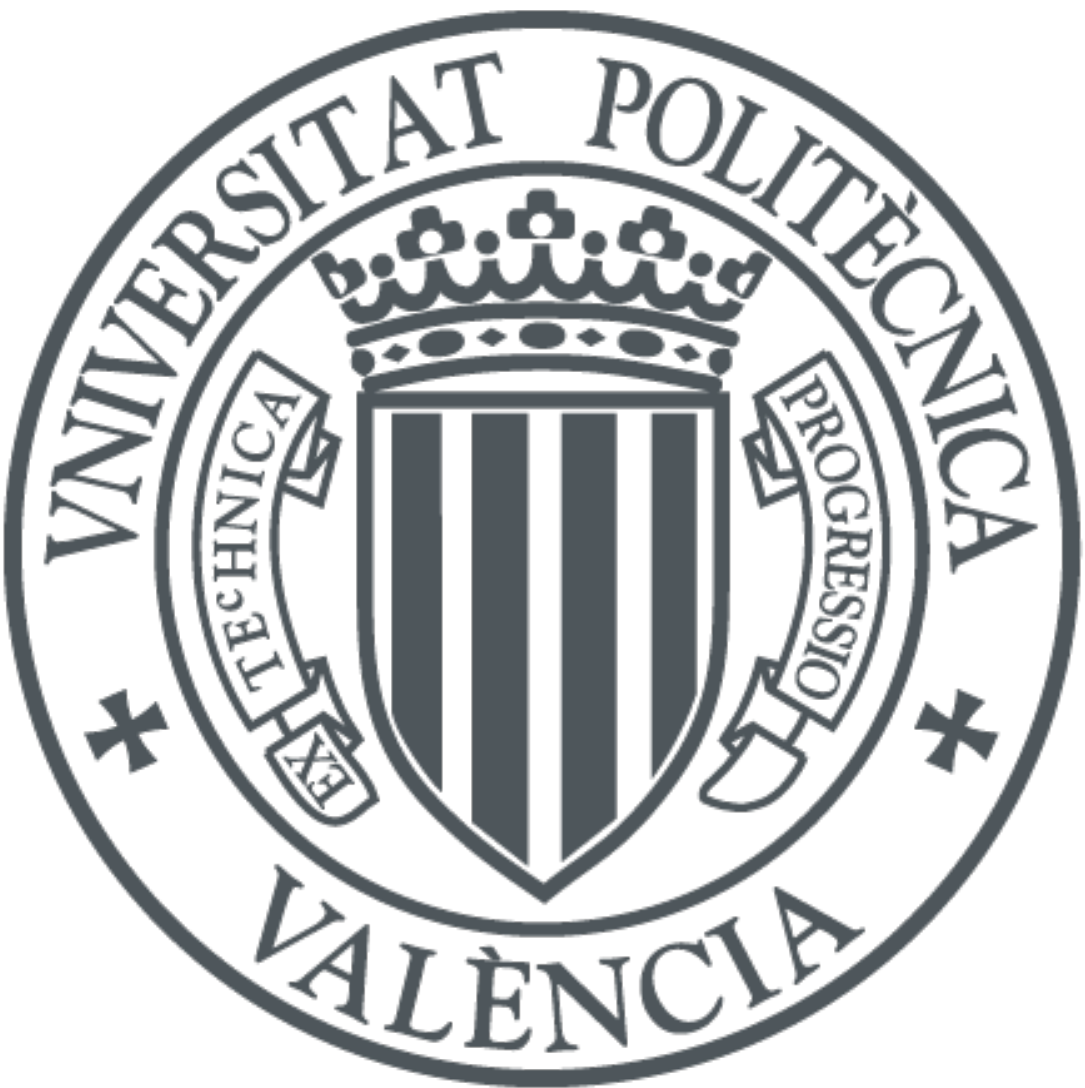

The final publication is available at

https://doi.org/10.1016/j.anucene.2017.08.018

Copyright Elsevier

Additional Information 


\title{
Spatial modes for the neutron diffusion equation and their computation
}

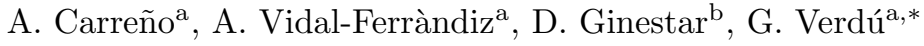 \\ ${ }^{a}$ Instituto de Seguridad Industrial, Radiofísica y Medioambiental, \\ Universitat Politècnica de València, Camino de Vera, $s / n$, 46022, Valencia \\ ${ }^{b}$ Instituto Universitario de Matemática Multidisciplinar, \\ Universitat Politècnica de València, Camino de Vera, s/n, 46022, Valencia
}

\begin{abstract}
Different spatial modes can be defined for the neutron diffusion equation such as the $\lambda, \alpha$ and $\gamma$-modes. These modes have been successfully used for the analysis of nuclear reactor characteristics. In this work, these modes are studied using a high order finite element method to discretize the equations and also different methods to solve the resulting algebraic eigenproblems, are compared. Particularly, Krylov subspace methods and block-Newton methods have been studied. The performance of these methods has been tested in several 3D benchmark problems: a homogeneous reactor and several configurations of NEACRP reactor.
\end{abstract}

Keywords: Spatial modes, Finite element, Neutron diffusion equation, Block Newton method, Generalized eigenvalue problem

\section{Introduction}

Different kind of spatial modes have been defined for the neutron transport equation forcing the criticality of the system under study by modifying the cross-sections in different ways (Bell \& Glasstone, 1970; Henry, 1975; Ronen 5 et al., 1976), obtaining different eigenvalue problems known as the $\lambda$-modes, the $\alpha$-modes and $\gamma$-modes. In Ronen et al. (1976); Velarde et al. (1978) these different modes are discussed and compared for fast neutron plutonium systems.

The multigroup diffusion equation is generally used to study reactor cores. These spatial modes can be also defined for this approximation and used for different purposes. In this way, the dominant $\lambda$-modes can be efficiently computed (Verdú et al., 1994), (Verdú et al., 1999), and they have been used to study and classify the neutronic oscillations in BWR reactors (March-Leuba \& Blakeman,

\footnotetext{
* Corresponding author

Email addresses: amcarsan@iqn.upv.es (A. Carreño), anvifer2@upv.es (A. Vidal-Ferràndiz), dginesta@mat.upv.es (D. Ginestar), gverdu@iqn.upv.es (G. Verdú)
} 
1991), (Verdú et al., 1998), (Miró et al., 2000) and to develop modal methods to solve the time dependent neutron diffusion equation (Miró et al., 2002).

The $\alpha$-modes problem is basic in the field of nuclear reactor physics (Bell \& Glasstone, 1970). These modes are important to develop monitoring techniques for subcritical systems (Lewins, 2013), (Kópházi \& Lathouwers, 2012), (Uyttenhove et al., 2014). Efficient methods have been developed for the computation of the dominant $\alpha$-modes of a reactor core using neutron diffusion equation

20 (Modak \& Gupta, 2007), (Verdu et al., 2010), (Singh et al., 2011) and also using neutron transport (Lathouwers, 2003), (Gupta \& Modak, 2011).

The $\gamma$-modes were presented in (Ronen et al., 1976) and (Velarde et al., 1978), but as far as the authors know, there is not a lot of work devoted to this kind of modes. Recently, (Avvakumov et al., 2017) a new spectral problem has 25 been formulated ( $\delta$-modes), which is connected to self-adjoint part of operator of neutron absorption-generation to make an a priori estimate of neutron flux dynamics.

Different methods have been proposed to discretize the neutron diffusion equation. Modern nodal methods usually rely in the Nodal Expansion Method

30 (NEM) (Finnemann; Singh et al., 2014) and analytical nodal method (ANM) (Smith, 1979; Hébert, 1987). Also, nodal collocation methods have been used to study reactors with rectangular geometries (Verdú et al., 1994). $h-p$ high order finite elements methods have also developed using two refinement techniques: a subdivision of the spatial mesh ( $h$-refinement) and also the possibility 35 of increasing the polynomial degree used in the finite element expansions (Wang et al., 2009). In this work, to discretize the different modes equations, a high order finite element method similar to the one presented in (Vidal-Ferrandiz et al., 2014) is used.

Generally, the dominant (or the smallest) eigenvalue and its corresponding 40 eigenfunction are computed to study the criticality of reactor and to know the steady state neutron distribution in the core. Next eigenvalues are interesting because they have been successfully used to develop modal methods and to classify and study neutronic oscillations (Miró et al., 2002). Thus, it will be interesting to develop efficient methods to compute a set of eigenpairs, not just

45 the first one. For that purpose, Krylov subspace based methods have shown effective, (Verdú et al., 1999), (Verdú et al., 2005). Nevertheless, to compute the dominant modes of a reactor for different configurations, block-Newton methods have shown to be very efficient (Lösche et al., 1998), (González-Pintor et al., 2011). These authors have studied a block-Newton method to solve ordinary eigenvalue problems. Since the different modes equations are expressed as generalized eigenvalue problems, in this work, two new extensions of the block-Newton method for generalized eigenvalue problems are proposed.

The main aim of this paper is to obtain, using a finite element method, the discrete eigenvalue problems associated with the $\lambda, \gamma$ and $\alpha$-modes, of the 55 neutron diffusion equation to compare these modes and to analyze different strategies to compute them for a given configuration of a nuclear power reactor, combining the Krylov-Schur method (Stewart, 2002) and the block-Newton methods for generalized eigenvalue problems. 
The structure of the rest of the paper is as follows. In section 2 , the $\lambda, \gamma$ and $\alpha$ modes are defined for the neutron diffusion equation in the approximation of two energy groups. Also, in this section, the relations between these modes are analyzed. In section 3, the spatial discretization used for the modes equations is briefly presented. In section 4 , the description of eigensolvers used to solve the algebraic problems obtained with the discretization is given. Numerical 65 results for the analysis of two different kind of reactors are presented in section 5. Finally, the main conclusions of the paper are summarized in section 6 .

\section{Definition of spatial modes}

The spatial modes problems are obtained from the two energy groups approximation of the neutron diffusion equation, however the formulations obtained can be easily extended to any number of groups of energy.

The time dependent neutron diffusion equation with $K$ groups of delayed neutron precursors is of the form (Stacey, 2007)

$$
\begin{aligned}
& V^{-1} \frac{\partial \phi}{\partial t}+\mathcal{L} \phi+\mathcal{S} \phi=(1-\beta) \mathcal{F} \phi+\sum_{k=1}^{K} \lambda_{k}^{d} \mathcal{C}_{k} \chi, \\
& \frac{\mathrm{d} \mathcal{C}_{k}}{\mathrm{~d} t}=\beta_{k} \mathcal{F}_{1} \phi-\lambda_{k}^{d} \mathcal{C}_{k}, \quad k=1, \ldots, K,
\end{aligned}
$$

where

$$
\begin{aligned}
& \mathcal{L}=\left(\begin{array}{cc}
-\vec{\nabla}\left(D_{1} \vec{\nabla}\right)+\Sigma_{a_{1}}+\Sigma_{12} & 0 \\
0 & -\vec{\nabla}\left(D_{2} \vec{\nabla}\right)+\Sigma_{a_{2}}
\end{array}\right), \\
& \mathcal{S}=\left(\begin{array}{cc}
0 & 0 \\
-\Sigma_{12} & 0
\end{array}\right), \quad \mathcal{F}=\left(\begin{array}{cc}
\nu \Sigma_{f_{1}} & \nu \Sigma_{f_{2}} \\
0 & 0
\end{array}\right), \\
& V^{-1}=\left(\begin{array}{cc}
\frac{1}{v_{1}} & 0 \\
0 & \frac{1}{v_{2}}
\end{array}\right), \quad \chi=\left(\begin{array}{c}
1 \\
0
\end{array}\right), \quad \phi=\left(\begin{array}{c}
\phi_{1} \\
\phi_{2}
\end{array}\right), \\
& \mathcal{F}_{1}=\left(\begin{array}{ll}
\nu \Sigma_{f_{1}} & \nu \Sigma_{f_{2}}
\end{array}\right) .
\end{aligned}
$$

Equation (1) can be transformed into several time-independent eigenvalue problems. Thus, criticality is forced of several forms obtained different eigenvalue problems: the $\lambda$ and the $\gamma$-modes problems. Assuming that the time dependence of the neutron flux has an exponential behaviour the $\alpha$-modes problem 75 is obtained.

If the fission nuclear cross sections are divided by a positive number, $\lambda$, the following steady-state equations are obtained

$$
\begin{aligned}
& \mathcal{L} \psi+\mathcal{S} \psi=(1-\beta) \frac{\mathcal{F}}{\lambda} \psi+\sum_{k=1}^{K} \lambda_{k}^{d} \mathcal{C}_{k} \chi, \\
& 0=\beta_{k} \frac{\mathcal{F}}{\lambda} \psi-\lambda_{k}^{d} \mathcal{C}_{k} \chi, \quad k=1, \ldots, K,
\end{aligned}
$$


that is,

$$
\mathcal{L} \psi+\mathcal{S} \psi=(1-\beta) \frac{\mathcal{F}}{\lambda} \psi+\sum_{k=1}^{K} \beta_{k} \frac{\mathcal{F}}{\lambda} \psi .
$$

Taking into account that $\sum_{k=1}^{K} \beta_{k}=\beta$, the $\lambda$-modes problem is obtained,

$$
(\mathcal{L}+\mathcal{S}) \psi_{n}=\frac{1}{\lambda_{n}} \mathcal{F} \psi_{n}
$$

The adjoint problem associated with the $\lambda$-modes is given by

$$
\left(\mathcal{L}^{\dagger}+\mathcal{S}^{\dagger}\right) \psi_{n}^{\dagger}=\frac{1}{\lambda_{n}} \mathcal{F}^{\dagger} \psi_{n}^{\dagger},
$$

where $\mathcal{L}^{\dagger}, \mathcal{S}^{\dagger}$ and $\mathcal{F}^{\dagger}$ are the transpose operators of $\mathcal{L}, \mathcal{S}$ and $\mathcal{F}$, respectively.

The $\lambda$-modes, $\psi_{n}$, and the adjoint $\lambda$-modes, $\psi_{m}^{\dagger}$, satisfy the biorthogonality relation

$$
\left\langle\psi_{m}^{\dagger}, \mathcal{F} \psi_{n}\right\rangle=\int_{\Omega} d V\left(\psi_{m}^{\dagger}\right)^{T} \mathcal{F} \psi_{n}=\delta_{m, n}\left\langle\psi_{n}^{\dagger}, \mathcal{F} \psi_{n}\right\rangle,
$$

where $\Omega$ is the volume defined by the reactor core and $\delta_{m, n}$ is the Kronecker's delta.

If the fission and scattering terms of (1) are divided by $\gamma>0$ to obtain the steady-state equations, a process similar to the one used to obtain the $\lambda$-modes can be followed to obtain the $\gamma$-modes problem, which has the following form

$$
\mathcal{L} \phi_{n}=\frac{1}{\gamma_{n}}(\mathcal{F}-\mathcal{S}) \phi_{n} .
$$

It is possible to obtain a relation between the $\lambda$-modes and the $\gamma$-modes in terms of the adjoint $\lambda$-modes problem (6). We start multiplying the equation (8) by the adjoint $\lambda$-mode, $\psi_{n}^{\dagger}$, and integrating over the domain, obtaining

$$
\left\langle\psi_{n}^{\dagger}, \mathcal{L} \phi_{n}\right\rangle=\left\langle\psi_{n}^{\dagger}, \frac{1}{\gamma_{n}}(\mathcal{F}-\mathcal{S}) \phi_{n}\right\rangle,
$$

or by symmetry of $\mathcal{L}$,

$$
\left\langle\mathcal{L} \psi_{n}^{\dagger}, \phi_{n}\right\rangle=\left\langle\psi_{n}^{\dagger}, \frac{1}{\gamma_{n}}(\mathcal{F}-\mathcal{S}) \phi_{n}\right\rangle .
$$

By equation (6),

$$
\mathcal{L} \psi_{n}^{\dagger}=\frac{1}{\lambda_{n}} \mathcal{F}^{\dagger} \psi_{n}^{\dagger}-\mathcal{S}^{\dagger} \psi_{n}^{\dagger},
$$

thus, equation (10) is equivalent to

$$
\frac{1}{\lambda_{n}}\left\langle\mathcal{F}^{\dagger} \psi_{n}^{\dagger}, \phi_{n}\right\rangle=\frac{1}{\gamma_{n}}\left\langle\psi_{n}^{\dagger},(\mathcal{F}-\mathcal{S}) \phi_{n}\right\rangle+\left\langle\psi_{n}^{\dagger}, \mathcal{S} \phi_{n}\right\rangle .
$$


Simplifying and isolating $\lambda_{n}$ in (12), we have

$$
\frac{1}{\lambda_{n}}=\frac{1}{\gamma_{n}}+\left(1-\frac{1}{\gamma_{n}}\right) \frac{\left\langle\psi_{n}^{\dagger}, \mathcal{S} \phi_{n}\right\rangle}{\left\langle\psi_{n}^{\dagger}, \mathcal{F} \phi_{n}\right\rangle} .
$$

To obtain the intermediate $\alpha$-modes, we consider again the neutron diffusion equation (1) where the delayed neutron precursors are assumed to be in steady state, that is

$$
0=\beta_{k} \mathcal{F}_{1} \phi-\lambda_{k}^{d} \mathfrak{C}_{k}, \quad k=1, \ldots, K .
$$

Other treatment of the neutron precursors lead to the prompt or total $\alpha$-modes

(Verdu et al., 2010).

This equality and the definition of $\beta$ imply that

$$
V^{-1} \frac{\partial \phi}{\partial t}+\mathcal{L} \phi+\mathcal{S} \phi=\mathcal{F} \phi .
$$

Assuming that the neutronic flux admits a factorization

$$
\phi(\vec{r}, t)=e^{\alpha t} \varphi(\vec{r}),
$$

we obtain the $\alpha$-modes equation

$$
(-V(\mathcal{L}+\mathcal{S})+V \mathcal{F}) \varphi=\alpha \varphi .
$$

The relation between the $\alpha$ - modes and the $\lambda$-modes is given by (Verdu et al., 2010)

$$
\alpha_{n}=\left(1-\frac{1}{\lambda_{n}}\right) \frac{\left\langle\psi_{n}^{\dagger}, \mathcal{F} \varphi_{n}\right\rangle}{\left\langle\psi_{n}^{\dagger}, V^{-1} \varphi_{n}\right\rangle} .
$$

\section{Finite element method discretization}

For all the modes equations, the discretization used has been a high order finite element method. For simplicity, this discretization is briefly reviewed only for the $\lambda$-modes problem in one group of energy but the same process can be applied to the $\gamma$ and $\alpha$-modes problems and to problems with more groups of energy.

Let us consider the $\lambda$-modes equation be for one group of energy,

$$
\left(-\vec{\nabla}(D \vec{\nabla})+\Sigma_{a}\right) \psi=\frac{1}{\lambda}\left(\nu \Sigma_{f}\right) \psi .
$$

The weak formulation is

$$
\int_{\Omega} \Phi\left(-\vec{\nabla}(D \vec{\nabla})+\Sigma_{a}\right) \psi \mathrm{d} V=\frac{1}{\lambda} \int_{\Omega} \Phi\left(\nu \Sigma_{f}\right) \psi \mathrm{d} V,
$$

where $\Phi$ is a test function and $\Omega$ the reactor domain. 
It is supposed that $\Omega$ can be decomposed as

$$
\Omega=\bigcup_{e=1}^{N_{t}} \Omega_{e},
$$

where the cross sections in $\Omega_{e}$ remain constant for all $e=1, \ldots, N_{t}$.

The solution $\psi$ is approximated in each node $\Omega_{e}$ as sum of shape functions $N_{j}$, which are Lagrange polynomials, multiplied by their corresponding nodal values $\tilde{\psi}_{j, e}$, as,

$$
\psi \approx \sum_{j=1}^{(p+1)^{d}} N_{j} \tilde{\psi}_{j, e},
$$

where $p$ is the degree of the polynomial expansion considered in the finite element method and $d$ the dimension $d=1,2$ or 3 depending on the geometry $(1 \mathrm{D}, 2 \mathrm{D}$ or $3 \mathrm{D})$ of the problem.

Introducing the assumptions (21) and (22) in the weak formulation (20) and using the Gauss Divergence theorem, an approximation of (19) can be computed solving the generalized algebraic eigenvalue problem

$$
\mathbf{A} \tilde{\psi}=\frac{1}{\lambda} \mathbf{B} \tilde{\psi},
$$

where the matrix elements are given by

$$
\begin{aligned}
A_{i j} & =\sum_{e=1}^{N_{t}}\left(D \int_{\Omega_{e}} \vec{\nabla} N_{i} \cdot \vec{\nabla} N_{j} \mathrm{~d} V-D \int_{\Gamma_{e}} N_{i} \vec{\nabla} N_{j} \mathrm{~d} \vec{S}+\Sigma_{a} \int_{\Omega_{e}} N_{i} N_{j} \mathrm{~d} V\right), \\
B_{i j} & =\sum_{e=1}^{N_{t}} \nu \Sigma_{f} \int_{\Omega_{e}} N_{i} N_{j} \mathrm{~d} V .
\end{aligned}
$$

The finite element method admits zero-flux, zero-current and mixed albedo boundary conditions. For more details see (Vidal-Ferrandiz et al., 2014). This method has been implemented using the open source finite elements library 95 Deal.II (Bangerth et al., 2007).

\section{Eigenvalue solvers}

Different generalized eigenvalue problems are obtained from the discretization for each type of modes problem. A set of dominant (largest in magnitude) eigenvalues and their corresponding eigenfunctions have to be computed for the $\lambda$ and $\gamma$-modes problems. For the $\alpha$-modes we are interested in the eigenvalues closest to zero (Verdu et al., 2010).

For two energy groups, the algebraic $\lambda$-modes problem has the form

$$
A^{\lambda} \psi=\frac{1}{\lambda} B^{\lambda} \psi,
$$


Matrices $A^{\lambda}$ and $B^{\lambda}$ have the following block structure

$$
A^{\lambda}=\left(\begin{array}{cc}
A_{11}^{\lambda} & 0 \\
A_{21}^{\lambda} & A_{22}^{\lambda}
\end{array}\right), \quad B^{\lambda}=\left(\begin{array}{cc}
B_{11}^{\lambda} & B_{12}^{\lambda} \\
0 & 0
\end{array}\right),
$$

so the problem (25) can be reduced to an ordinary eigenvalue problem

$$
\left(A_{11}^{\lambda}\right)^{-1}\left(B_{11}^{\lambda}-B_{12}^{\lambda}\left(A_{22}^{\lambda}\right)^{-1} A_{21}^{\lambda}\right) \tilde{\psi}_{1}=\lambda \tilde{\psi}_{1},
$$

where the size of the associated matrix is one half of the size of the matrices of the generalized eigenvalue problem.

For the $\gamma$ and $\alpha$-modes problems this kind of reduction is not possible. So, two possibilities have been considered for their computation. First, the generalized problems are reduced to an ordinary eigenvalue problem and this problem is solved using Krylov-Schur method. Then, for the $\gamma$-modes, the generalized eigenvalue problem associated is

$$
A^{\gamma} \phi=\frac{1}{\gamma} B^{\gamma} \phi
$$

with

$$
A^{\gamma}=\left(\begin{array}{cc}
A_{11}^{\gamma} & 0 \\
0 & A_{22}^{\gamma}
\end{array}\right), \quad B^{\gamma}=\left(\begin{array}{cc}
B_{11}^{\gamma} & B_{12}^{\gamma} \\
B_{21}^{\gamma} & 0
\end{array}\right),
$$

which is reduced to

$$
\left(\mathbf{A}^{\gamma}\right)^{-1} \mathbf{B}^{\gamma} \tilde{\phi}=\gamma \tilde{\phi} .
$$

The problem obtained for $\alpha$-modes is

$$
A^{\alpha} \varphi=\alpha B^{\alpha} \varphi
$$

where their matrices have the following structure

$$
A^{\alpha}=\left(\begin{array}{ll}
A_{11}^{\alpha} & A_{12}^{\alpha} \\
A_{21}^{\alpha} & A_{22}^{\alpha}
\end{array}\right), \quad B^{\alpha}=\left(\begin{array}{cc}
B_{11}^{\alpha} & 0 \\
0 & B_{22}^{\alpha}
\end{array}\right) .
$$

Note that although the equation (17) corresponds to continuous ordinary eigenvalue problem, since the finite element basis used for the spatial discretization is not orthogonal, the discrete eigenvalue problem obtained is a generalized eigenvalue problem, where the matrix $B^{\alpha}$ essentially corresponds to the massmatrix of the finite element method.

The generalized eigenvalue problem for $\alpha$-modes problem can be reduced to

$$
\left(\mathbf{A}^{\alpha}\right)^{-1} \mathbf{B}^{\gamma} \tilde{\varphi}=\tilde{\alpha} \tilde{\varphi}, \text { with } \tilde{\alpha}=\frac{1}{\alpha} .
$$

The matrix inverses do not need to be computed explicitly. They can be handled implicitly by solving systems of linear equations. These systems are solved by BiCGStab method, together a Cuthill-McKee reordering and incomplete LU factorization for preconditioning the matrices (Saad, 2003). 
The second possibility, is to solve the $\lambda$-modes problem, which is the easiest problem to be solved and use these modes to initialize a block-Newton method to solve the $\alpha$ and $\gamma$ generalized eigenvalue problems. For the $\gamma$-modes problem we have

$$
\mathbf{B}^{\gamma} \tilde{\phi}=\gamma \mathbf{A}^{\gamma} \tilde{\phi},
$$

and for $\alpha$-modes it is

$$
\mathbf{B}^{\alpha} \tilde{\varphi}=\tilde{\alpha} \mathbf{A}^{\alpha} \tilde{\varphi}, \text { with } \tilde{\alpha}=\frac{1}{\alpha} .
$$

With this formulation all problems (ordinary and generalized) are solved for the $q$ dominant eigenvalues and their corresponding eigenvectors. Moreover, the eigenvectors are normalized fixing the mean power production to 1, that is,

$$
\bar{P}=\frac{1}{V_{t}} \int_{\Omega}\left(\kappa_{1} \Sigma_{f 1}\left|\xi_{1}\right|+\kappa_{2} \Sigma_{f 2}\left|\xi_{2}\right|\right) \mathrm{d} V=1,
$$

where $V_{t}$ is the volume of the reactor core, and $\xi_{i}, i=1,2$ are the corresponding fast and thermal eigenfunctions. $\kappa_{1}$ and $\kappa_{2}$ denote the energies per fission for fast and thermal groups. The absolute value is introduced because the 'subcritical' eigenfunctions are not positive in the whole reactor domain.

\subsection{Krylov-Schur method}

The Krylov-Schur method was introduced in 2002 by Stewart (Stewart, 2002) and can be seen as an improvement on traditional Krylov subspace methods such as Arnoldi and Lanczos for computing a subset of eigenvalues and their corresponding eigenvectors of a large and sparse matrix. The basic idea of the Krylov-Schur method is to iteratively expand (with the Arnoldi process) and contract with a so-called Krylov-Schur decomposition. To solve the ordinary and generalized eigenvalue problems by Krylov-Schur method, the library SLEPc (Hernandez et al., 2005) has been used.

\subsection{Block-Newton method}

To compute the $\alpha$ and $\gamma$ modes it is interesting to take into account that the space spanned by these modes, near the criticality, is close to the space spanned by the $\lambda$-modes. For this reason, a Modified block-Newton method is applied to 130 compute the $\alpha$ and $\gamma$-modes using as initial guess the $\lambda$-modes. In the following, the main ideas of this method are exposed.

\subsubsection{Initial approximation}

First, a procedure based on Rayleigh-Ritz method is applied to obtain an initial approximation for the solution of $\gamma$ and $\alpha$-modes.

We start with a partial ordinary eigenvalue problem

$$
M_{\delta} V_{\delta}=V_{\delta} \Lambda_{\delta}, \quad \delta=\alpha, \gamma,
$$

135

where $M_{\delta} \in \mathbb{R}^{n \times n}$ is the matrix of the ordinary eigenvalue problem associated with the $\alpha$ and $\gamma$ problems, $V_{\delta} \in \mathbb{R}^{n \times q}$ is a matrix with $q$ eigenvectors associated 
with the $q$ dominant eigenvalues of the $\gamma$ or $\alpha$-modes problem, defined in equations (34) and (35), respectively, and $\Lambda_{\delta}$ is a diagonal matrix whose elements are the dominant eigenvalues.

It is assumed that the eigenvectors for the $\gamma$ and $\alpha$-modes problems can be approximately expressed as a linear combination of $\lambda$-modes eigenvectors,

$$
V_{\delta}=V_{\lambda} Z, \text { with } V_{\lambda}^{T} V_{\lambda}=I .
$$

Then the problem (37) can be approximately rewritten as

$$
\left(V_{\lambda}^{T} M_{\delta} V_{\lambda}\right) Z=Z \tilde{\Lambda}_{\delta}
$$

140 values $q$, which is much lower than $n$, the size of $M_{\delta}$, thus, this problem can be easily solved using a direct method. The eigenvectors $Z$ and the corresponding eigenvalues given by $\tilde{\Lambda}_{\delta}$ are the Ritz pairs of $M_{\delta}$ and matrix $V_{\delta}$ defined by (38) can be used as an initial approximation of the eigenvectors of original problem 145 (37) using a block-Newton method for ordinary eigenvalue problems (Lösche et al., 1998; González-Pintor et al., 2011).

Nevertheless, $\gamma$ and $\alpha$-modes problems are expressed in terms of generalized eigenvalue problems (34) and (35). To compute the solution of these problems reducing them to an ordinary eigenvalue problems presents numerical precision problems as it will be discussed below. In this way, to solve the generalized eigenvalue problems for $\gamma$ and $\alpha$ modes we propose two new generalizations of the Modified block-Newton method for generalized eigenvalue problems.

\subsubsection{Modified block-Newton method with generalized Rayleigh-Ritz (MBNM- $G R R$ )}

Given a generalized eigenvalue problem of the form

$$
A V=B V \Lambda,
$$

where $V \in \mathbb{R}^{n \times q}$ is a matrix of eigenvectors and $\Lambda \in \mathbb{R}^{q \times q}$ is a diagonal matrix whose diagonal elements are the dominant eigenvalues. It is assumed that the eigenvectors can be factorized as

$$
V=Z S
$$

where $Z^{\mathrm{T}} Z=I_{q}$. Problem (40) can be rewritten as

$$
A V=B V \Lambda \Rightarrow A Z S=B Z S \Lambda \Rightarrow A Z=B Z S \Lambda S^{-1} \Rightarrow A Z=B Z K .
$$

This problem is undetermined since the eigenvectors are defined up to a constant. To determine the problem, the biorthogonality condition $W^{\mathrm{T}} Z=I_{q}$ is introduced, where $W$ is a fixed matrix of rank $q$. Newton's method is used to solve the following problem

$$
F(Z, \Lambda):=\left(\begin{array}{c}
A Z-B Z K \\
W^{\mathrm{T}} Z-I_{q}
\end{array}\right)=\left(\begin{array}{l}
0 \\
0
\end{array}\right) .
$$


Thus, a new iterated solution arises as,

$$
Z^{(k+1)}=Z^{(k)}-\Delta Z^{(k)}, \quad K^{(k+1)}=K^{(k)}-\Delta K^{(k)},
$$

where $\Delta Z^{(k)}$ and $\Delta K^{(k)}$ are solutions of the system

$$
\left\{\begin{array}{l}
A \Delta Z^{(k)}-B \Delta Z^{(k)} K^{(k)}-B Z^{(k)} \Delta K^{(k)}=A Z^{(k)}-B Z^{(k)} K^{(k)}, \\
W^{T} \Delta Z^{(k)}=W^{T} Z^{(k)}-I_{q}
\end{array}\right.
$$

that is obtained substituting (44) into (43) and removing second order terms.

The system (45) is coupled, since the matrix $K$ is not necessarily diagonal. To decouple the system, the Modified block-Newton method applies two previous steps. The first step consists of an orthogonalization to the matrix $Z^{(k)}$ using the modified Gram-Schmidt Orthogonalization. Once $Z^{(k)}$ is an orthonormal matrix, i.e., $Z^{(k)^{\mathrm{T}}} Z^{(k)}=I_{q}$, as a second step, a Rayleigh-Ritz procedure for generalized eigenvalue problems is applied (Saad, 1992; Li, 2014), which consists of obtaining the eigenvectors $S^{(k)}$ and their corresponding eigenvalues $\Lambda^{(k)}$ that satisfy

$$
Z^{(k)^{\mathrm{T}}} A Z^{(k)} S^{(k)}=Z^{(k)^{\mathrm{T}}} B Z^{(k)} S^{(k)} \Lambda^{(k)} .
$$

Defining $\bar{Z}^{(k)}:=Z^{(k)} S^{(k)}$, we have, from (46), that $\Lambda^{(k)}$ is a diagonal matrix whose elements, $\lambda_{i}$ are the Ritz values and $\bar{Z}^{(k)}$ are the approximated Ritz eigenvectors, satisfying the equation

$$
Z^{(k)^{\mathrm{T}}}\left(A \bar{Z}^{(k)}-B \bar{Z}^{(k)} \Lambda^{(k)}\right)=0 .
$$

At each iteration, the matrix $W$ in equation (45) is chosen as the previous approximation for the invariant subspace, that is, $W=\bar{Z}^{(k)}$. Using the definition of $K^{(k)}$ on (42), system (45) is decoupled into the $q$ linear systems

$$
\left(\begin{array}{cc}
A-B \lambda_{i}^{(k)} & B \bar{Z}^{(k)} \\
\bar{Z}^{(k)^{\dagger}} & 0
\end{array}\right)\left(\begin{array}{c}
\Delta \bar{z}_{i}^{(k)} \\
-\Delta \lambda_{i}^{(k)}
\end{array}\right)=\left(\begin{array}{c}
A \bar{z}_{i}^{(k)}-B \bar{z}_{i}^{(k)} \lambda_{i}^{(k)} \\
0
\end{array}\right), i=1, \ldots, q,
$$

where $\Delta \bar{z}_{i}^{(k)}$ is the $i$-th column of $\Delta \bar{Z}^{(k)}$. Vectors $Z^{(k+1)}$ are updated according to equation (44) and the eigenvalues $\lambda_{i}^{(k)}$ are obtained from the small problem (46).

\subsubsection{Modified block-Newton method with biorthogonalization process (MBNM-} $G B O)$

Given the generalized eigenvalue problem (40), as in the previous method, the eigenvectors are expressed as

$$
V=Z S
$$

but now vectors $Z$ are chosen to satisfy $H^{\mathrm{T}} B Z=I_{q}$ for some $H \in \mathbb{R}^{n \times q}$. Problem (40) is rewritten as

$$
A Z=B Z K
$$


and to determine the problem, now the biorthogonality condition $W^{T} B Z=I_{q}$ is introduced, where $W$ is a fixed matrix of rank $q$, obtaining the problem

$$
F(Z, \Lambda):=\left(\begin{array}{c}
A Z-B Z K \\
W^{\mathrm{T}} B Z-I_{q}
\end{array}\right)=\left(\begin{array}{l}
0 \\
0
\end{array}\right) .
$$

From Newton's method, a new iterated solution arises as

$$
Z^{(k+1)}=Z^{(k)}-\Delta Z^{(k)}, \quad K^{(k+1)}=K^{(k)}-\Delta K^{(k)},
$$

where $\Delta Z^{(k)}$ and $\Delta K^{(k)}$ are solutions of the system

$$
\left\{\begin{array}{l}
A \Delta Z^{(k)}-B \Delta Z^{(k)} K^{(k)}-B Z^{(k)} \Delta K^{(k)}=A Z^{(k)}-B Z^{(k)} K^{(k)}, \\
W^{T} B \Delta Z^{(k)}=W^{T} B Z^{(k)}-I_{q} .
\end{array}\right.
$$

To decouple system (53), the Modified block-Newton method applies two previous steps. The first step is to apply to matrix $Z^{(k)}$ an algorithm, based on a biorthogonalization process (Adrover et al., 2005), to obtain $Z^{(k)}$ and $H^{(k)}$ such that $H^{(k)^{\mathrm{T}}} B Z^{(k)}=I_{q}$, (see Algorithm 1), where $H^{(k)}$ is initiated as $Z^{(k)}$.

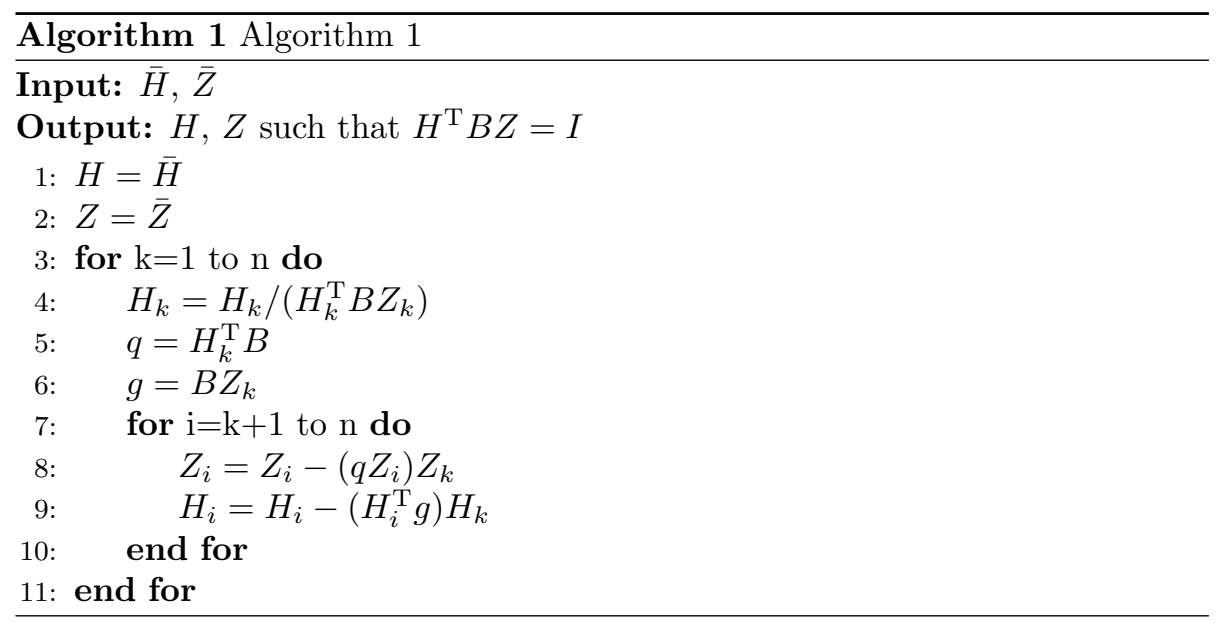

Once $Z^{(k)}$ and $H^{(k)}$ have been obtained, as a second step, a Rayleigh-Ritz procedure is applied, which consists of obtaining the eigenvectors $S^{(k)}$ and their corresponding eigenvalues $\Lambda^{(k)}$ that satisfy

$$
A Z^{(k)} S^{(k)}=B Z^{(k)} S^{(k)} \Lambda .
$$

Making use of the relation of matrix $Z^{(k)}$ and $H^{(k)}$, the following equation is obtained

$$
H^{(k)^{\mathrm{T}}} A Z^{(k)} S^{(k)}=S^{(k)} \Lambda^{(k)},
$$

which is a small generalized eigenvalue problem.

Defining $\bar{Z}^{(k)}:=Z^{(k)} S^{(k)}$, it is satisfied that

$$
H^{(k)^{\mathrm{T}}}\left(A \bar{Z}^{(k)}-B \bar{Z}^{(k)} \Lambda^{(k)}\right)=0 .
$$


At each iteration, the matrix $W$ is chosen as $W=H^{(k)}$, then the system (53) is decoupled into the $q$ linear systems

$$
\left(\begin{array}{cc}
A-B \lambda_{i}^{(k)} & B Z^{(k)} \\
H^{(k)^{\mathrm{T}}} B & 0
\end{array}\right)\left(\begin{array}{c}
\Delta z_{i}^{(k)} \\
-\Delta \lambda_{i}^{(k)}
\end{array}\right)=\left(\begin{array}{c}
A z_{i}^{(k)}-B z_{i}^{(k)} \lambda_{i}^{(k)} \\
0
\end{array}\right), i=1, \ldots, q .
$$

As in the previous method, only the eigenvectors are updated with $\Delta z_{i}^{(k)}$ and the eigenvalues are computed from the small problem (55).

\section{Numerical results}

To study and compare the different spatial modes of a nuclear reactor, different benchmarks problems in a 3D geometry have been considered. First, a 3D homogeneous reactor is studied and second, the NEACRP benchmark Finnemann \& Galati (1991) in different configurations are chosen to compare the different spatial modes and eigenvalue solvers in a more realistic case.

To analyze the methods used to compute the modes, different errors have been employed: the relative power error,

$$
\varepsilon_{i}=\frac{\left|P_{i}-P_{i}^{*}\right|}{\left|P_{i}\right|}
$$

the mean relative error,

$$
\bar{\varepsilon}=\frac{1}{V_{t}} \sum_{i} \varepsilon_{i} V_{i}
$$

and the eigenvalue error expressed in $p c m$,

$$
\varepsilon_{e i g}=10^{5}\left(\frac{\left|\delta_{i}-\delta_{i}^{*}\right|}{\left|\delta_{i}\right|}\right),
$$

where $P_{i}$ and $P_{i}^{*}$ are the reference power and the computed power in the $i$-th 175 cell (cell averages), respectively. $V_{i}$ is the volume of the cell and $V_{t}$ is the total volume of the reactor, $\delta_{i}$ and $\delta_{i}^{*}$ with $\delta=\lambda, \alpha, \gamma$ are the reference and computed eigenvalues.

Given a generalized egienvalue problem

$$
B^{\delta} v=\delta A^{\delta} v
$$

and the associated ordinary eigenvalue problem

$$
\left(A^{\delta}\right)^{-1} B^{\delta} v=\delta v,
$$

the following residuals have been considered:

$$
\operatorname{res}_{g}=\sqrt{\sum_{k=1, \ldots, q} \frac{\left\|B^{\delta} v_{k}-A^{\delta} v_{k} \delta_{k}\right\|^{2}}{\left|\delta_{k}\right|}}
$$


Table 1: Macroscopic cross section values for the homogeneous reactor.

\begin{tabular}{lllllll}
\hline$D_{1}\left(\mathrm{~cm}^{-1}\right)$ & $D_{2}\left(\mathrm{~cm}^{-1}\right)$ & $\Sigma_{a 1}\left(\mathrm{~cm}^{-1}\right)$ & $\Sigma_{a 2}\left(\mathrm{~cm}^{-1}\right)$ & $\Sigma_{12}\left(\mathrm{~cm}^{-1}\right)$ & $\nu \Sigma_{f 1}\left(\mathrm{~cm}^{-1}\right)$ & $\nu \Sigma_{f 2}\left(\mathrm{~cm}^{-1}\right)$ \\
\hline 1.5015 & $4.3290 \mathrm{e}-01$ & $9.4003 \mathrm{e}-03$ & $8.2108 \mathrm{e}-02$ & $1.6850 \mathrm{e}-02$ & $6.0600 \mathrm{e}-03$ & $1.0100 \mathrm{e}-01$ \\
\hline
\end{tabular}

and

$$
\operatorname{res}_{o}=\sqrt{\sum_{k=1, \ldots, q} \frac{\left\|\left(A^{\delta}\right)^{-1} B^{\delta} v_{k}-v_{k} \delta_{k}\right\|^{2}}{\left|\delta_{k}\right|}}
$$

where $q$ is the number of eigenvalues computed, $A^{\delta}$ and $B^{\delta}$ are the matrices of the $\delta$-modes problem, $\delta_{k}$ the $k$-th eigenvalue and $v_{k}$ its corresponding eigenvector with $\delta=\lambda, \gamma, \alpha$.

The computer used for the computations has been an Intel ${ }^{\circledR}$ Core $^{T M}$ i7-4790 @ $3.60 \mathrm{GHz} \times 8$ processor with $32 \mathrm{~Gb}$ of RAM running Ubuntu GNU/Linux 16.04 LTS.

\subsection{Homogeneous reactor}

A 3D prismatic reactor with homogeneous material is considered, since it can be solved analytically for all its eigenvalues and compared with the numerical results obtained using the finite element method. The dimensions considered are $300 \mathrm{~cm} \times 300 \mathrm{~cm} \times 450 \mathrm{~cm}$ and the material cross sections for the prismatic reactor are displayed in Table 5.1. The velocities are $v_{1}=2.8 \cdot 10^{7} \mathrm{~cm} / \mathrm{s}$ and $v_{2}=$

$1904.4 \cdot 10^{5} \mathrm{~cm} / \mathrm{s}$. The number of neutron produced by fission $(\nu)$ has been considered constant in the reactor core and equal to 2.5. The boundary conditions are zero flux at the boundary. The mesh considered for the discretization of the reactor is composed of 36 cells of size $50 \times 50 \mathrm{~cm} 2$ per 6 planes of height $75 \mathrm{~cm}$, having a total of 216 cells. The energies by fission $\left(\kappa_{1}\right.$ and $\left.\kappa_{2}\right)$ are set to $1 \mathrm{~J} /$ fission.

Table 2 shows the analytical and the numerical value of the eigenvalue computed with different finite element degrees for the first two $\lambda, \gamma$ and $\alpha$-modes. The method used to compute these modes has been Krylov-Schur that solves the ordinary eigenvalue problems, (27), (30) and (33). The number of eigenvalues requested has been $q=4$, the dimension of Krylov subspace chosen has been 19 and the relative tolerance has been set to res $_{o}=10^{-8}$. In these Tables we observe the convergence of the finite element method and that good approximations are obtained choosing a polynomial degree in the finite element method $p$ equal to 2 or larger. Furthermore, it is observed that for the same degree of polynomial the error in eigenvalues is lower for $\lambda$ and $\gamma$-modes than for $\alpha$-modes. The mean relative errors $(\bar{\varepsilon})$ obtained are negligible.

Computational data related to Krylov-Schur method are displayed in Table 5.1. The problem considered for each type of mode is the obtained from the spatial discretization using a polynomial degree equal to $p=3$ in the finite element method. If the iterations needed by the Krylov method to reach the same tolerance in the ordinary eigenvalue problem are compared, this value is 
Table 2: Modes and errors for the homogeneous reactor computed with Krylov-Schur method.

\begin{tabular}{cccccc}
\hline & \multicolumn{2}{c}{ First eigenvalue } & & \multicolumn{2}{c}{ Second eigenvalue } \\
\cline { 2 - 3 } \cline { 5 - 6 } $\mathbf{p}$ & $\boldsymbol{\delta}_{\mathbf{1}}$ & $\boldsymbol{\varepsilon}_{\boldsymbol{e} \text { eig }}(p c m)$ & & $\boldsymbol{\delta}_{\mathbf{2}}$ & $\boldsymbol{\varepsilon}_{\text {eig }}(p c m)$ \\
\hline $\boldsymbol{\lambda}$-modes & & & & \\
1 & 1.003649 & 37.27 & & 0.993831 & 140.29 \\
2 & 1.004022 & 0.12 & & 0.995208 & 1.98 \\
3 & 1.004023 & 0.02 & & 0.995227 & 0.01 \\
Anal. solut.: & 1.004024 & & & 0.995227 & \\
\hline $\boldsymbol{\gamma}$-modes & & & & \\
1 & 1.003619 & 134.92 & & 0.993831 & 348.44 \\
2 & 1.002266 & 0.07 & & 0.997295 & 1.10 \\
3 & 1.002267 & 0.02 & & 0.997306 & 0.01 \\
Anal. solut.: & 1.002266 & & 0.997306 & \\
\hline $\boldsymbol{\alpha}$-modes & & & & \\
1 & 160.6970 & 9313 & & -271.5494 & 29248 \\
2 & 177.1258 & 41 & & -210.9691 & 410 \\
3 & 177.1995 & 0.06 & & -210.1068 & 3.1 \\
Anal. solut.: & 177.1995 & & -210.1002 & \\
\hline
\end{tabular}

higher for $\gamma$-modes than the one obtained for other modes. This is due to the fact that the spectrum of these modes is more clustered. It can be seen in the dominance ratio of $\gamma_{1} / \gamma_{2}$. This property can be also observed for the other kind of modes, since the iterations of the Krylov method are larger when this ratio is closer to 1.

However, if we compare the mean number of the iterations to solve the linear systems with BiCGStab method, the lowest value is obtained for the $\gamma$-modes, since the matrix $A^{\gamma}$ is symmetric (see Eq. (29)). In this comparison, the mean number of iterations needed for the $\alpha$-modes is much larger when compared 220 with the other modes. The reason is that the matrix $A^{\alpha}$, that comes from the discretization of $-V(\mathcal{L}+\mathcal{S})+V \mathcal{F}$, is ill-conditioned.

We have estimated the condition number of each one of the matrices, $\operatorname{cond}\left(A^{\delta}\right)$, and this value for the matrix corresponding to the $\alpha$-modes problem is two orders of magnitude larger than the one for matrices corresponding to the other modes. Thus, it is very expensive to converge the solution of linear systems associated with $A^{\alpha}$. This is due to the quasi-criticality of the reactor, since in this case, the desired $\alpha$-modes are close to 0 and the matrices are ill-conditioned. Furthermore, the residual error obtained for the generalized problem, res $g$, for the $\alpha$-modes is very high $\left(\operatorname{res}_{g} \approx 1 e-2\right.$, as it is observed in Table 5.1) with respect to the error in the corresponding ordinary eigenvalue problem, that is res $_{o} \approx 1 e-8$. Then, in order to compute a solution of $\alpha$-modes with a residual error in the generalized eigenvalue problem $\operatorname{res}_{g} \approx 1 e-6$, we need to request approximately a tolerance in the corresponding ordinary eigenvalue problem of res $_{o} \approx 1 e-12$. With respect to the CPU times, the $\lambda$-modes are the cheapest 
Table 3: Data of eigenvalue problem for homogeneous reactor obtained with $p=3$ and 216 cells and computed with the Krylov-Schur method.

\begin{tabular}{cllcccl}
\hline Modes & $\begin{array}{l}\text { Its. } \\
\text { Krylov }\end{array}$ & $\begin{array}{l}\text { mean its. } \\
\text { BiCGStab }\end{array}$ & $\operatorname{cond}\left(A^{\delta}\right)$ & $\delta_{1} / \delta_{2}$ & $\operatorname{res} g$ & $\begin{array}{l}\text { CPU } \\
\text { Time(s) }\end{array}$ \\
\hline$\lambda$-modes & 13 & 6.99 & $2.59 \mathrm{e}+02$ & 1.009 & $3.75 \mathrm{e}-07$ & 3.1 \\
$\gamma$-modes & 23 & 4.00 & $2.13 \mathrm{e}+02$ & 1.005 & $4.17 \mathrm{e}-06$ & 12.8 \\
$\alpha$-modes & 3 & 30.48 & $5.71 \mathrm{e}+05$ & 0.843 & $6.85 \mathrm{e}-02$ & 15.3 \\
\hline
\end{tabular}

\subsection{NEACRP reactor}

A critical configuration of the NEACRP (case A1) benchmark (Finnemann \& Galati, 1991) is chosen to compare the different modes and eigenvalue solvers in a more realistic case. The core is composed of 221 assemblies including 64 cells modeling the reflector, with a radial dimension of $21.606 \mathrm{~cm} \times 21.606 \mathrm{~cm}$ per assembly. The definition of the different materials is shown in Figure 1. Axially the reactor, with the total height of $427.3 \mathrm{~cm}$, is divided into 18 layers with height (from bottom to top): $30.0 \mathrm{~cm}, 7.7 \mathrm{~cm}, 11.0 \mathrm{~cm}, 15.0 \mathrm{~cm}, 30.0$ $\mathrm{cm}$ (10 layers), $12.8 \mathrm{~cm}$ (2 layers), $8.0 \mathrm{~cm}$ and $30.0 \mathrm{~cm}$. The cross sections of 245 materials are displayed in Table 4 . The boundary condition for the solution is flux vanishing in the outer reflector surface. And the velocities are $v_{1}=2.8$. $10^{7} \mathrm{~cm} / \mathrm{s}$ and $v_{2}=4.4 \cdot 10^{5} \mathrm{~cm} / \mathrm{s}$. Some subcritical configurations are also defined dividing the fission cross sections of the different materials by 1.1 (Perturbation I) and by 1.2 (Perturbation II).

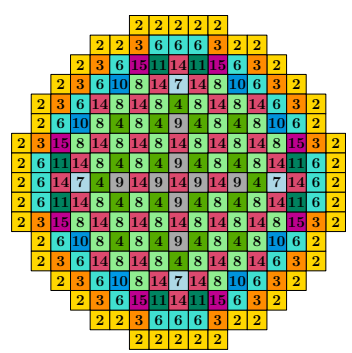

(a) $3-17$ th radial planes

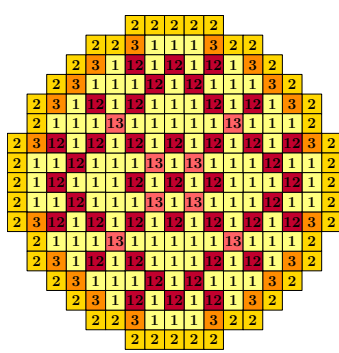

(b) 18th radial plane

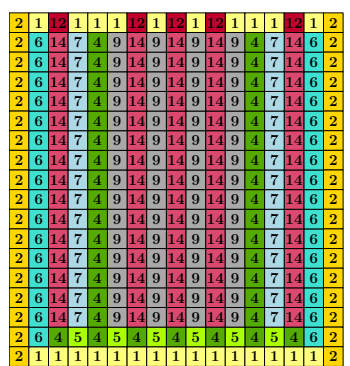

(c) Reactor profile

Figure 1: Geometry of NEACRP reactor in the critical state

In the following computations, the options used for the finite element method to discretize the different eigenvalue problems are: finite element degree $p=3$ and without refinement of the initial mesh. The size of the matrix (determined computing ndofs $\times$ ndofs where ndofs are the number of degrees of freedom) and the number of non-zero elements (nnz) are obtained. The results are displayed

255 in Table 5.2. In this Table, it is observed that ndofs for $\lambda$-modes problem is half the ndofs for $\gamma$ and $\alpha$-modes. This is due to the fact that the $\lambda$-modes eigenproblem can be solved as ordinary eigenvalue problem as the Eq. (27) and 
Table 4: Macroscopic cross section of the NEACRP reactor.

\begin{tabular}{|c|c|c|c|c|c|c|c|c|c|}
\hline Mat. & $D_{1}(\mathrm{~cm})$ & $D_{2}(\mathrm{~cm})$ & $\Sigma_{a 1}\left(\mathrm{~cm}^{-1}\right)$ & $\Sigma_{a 2}\left(\mathrm{~cm}^{-1}\right)$ & $\Sigma_{12}\left(\mathrm{~cm}^{-1}\right)$ & $\kappa_{1} \Sigma_{f 1}\left(\mathrm{Jcm}^{-1}\right)$ & $\kappa_{2} \Sigma_{f 2}\left(\mathrm{Jcm}^{-1}\right)$ & $\nu \Sigma_{f 1}\left(\mathrm{~cm}^{-1}\right)$ & $\nu \Sigma_{f 2}\left(\mathrm{~cm}^{-1}\right)$ \\
\hline 1 & 5.9264 & $8.2289 \mathrm{e}-01$ & $2.5979 \mathrm{e}-04$ & $1.7085 \mathrm{e}-01$ & $2.7988 \mathrm{e}-02$ & 0.0000 & 0.0000 & 0.0000 & 0.0000 \\
\hline 2 & 1.1276 & $1.7053 \mathrm{e}-01$ & $1.1878 \mathrm{e}-03$ & $1.9770 \mathrm{e}-01$ & $2.3161 \mathrm{e}-02$ & 0.0000 & 0.0000 & 0.0000 & 0.0000 \\
\hline 3 & 1.1276 & $1.7053 \mathrm{e}-01$ & $1.1878 \mathrm{e}-03$ & $1.9770 \mathrm{e}-01$ & $2.0081 \mathrm{e}-02$ & 0.0000 & 0.0000 & 0.0000 & 0.0000 \\
\hline 4 & 1.4624 & $3.9052 \mathrm{e}-01$ & $8.4767 \mathrm{e}-03$ & $6.2569 \mathrm{e}-02$ & $1.9686 \mathrm{e}-02$ & $6.1479 \mathrm{e}-14$ & $1.1515 \mathrm{e}-12$ & $5.0150 \mathrm{e}-03$ & $8.7712 \mathrm{e}-02$ \\
\hline 5 & 1.4637 & $3.9485 \mathrm{e}-01$ & $8.8225 \mathrm{e}-03$ & $6.9978 \mathrm{e}-02$ & $1.9436 \mathrm{e}-02$ & $6.9275 \mathrm{e}-14$ & $1.3685 \mathrm{e}-12$ & $5.6085 \mathrm{e}-03$ & $1.0424 \mathrm{e}-01$ \\
\hline 6 & 1.4650 & $3.9851 \mathrm{e}-01$ & $9.1484 \mathrm{e}-03$ & $7.6850 \mathrm{e}-02$ & $1.9196 \mathrm{e}-02$ & $7.6811 \mathrm{e}-14$ & $1.5694 \mathrm{e}-12$ & $6.1819 \mathrm{e}-03$ & $1.1954 \mathrm{e}-01$ \\
\hline 7 & 1.4641 & $4.0579 \mathrm{e}-01$ & $9.0869 \mathrm{e}-03$ & $7.7687 \mathrm{e}-02$ & $1.8526 \mathrm{e}-02$ & $6.8996 \mathrm{e}-14$ & $1.3509 \mathrm{e}-12$ & $5.5830 \mathrm{e}-03$ & $1.0289 \mathrm{e}-01$ \\
\hline 8 & 1.4642 & $4.0946 \mathrm{e}-01$ & $9.1738 \mathrm{e}-03$ & $8.0302 \mathrm{e}-02$ & $1.8223 \mathrm{e}-02$ & $6.8913 \mathrm{e}-14$ & $1.3433 \mathrm{e}-12$ & $5.5741 \mathrm{e}-03$ & $1.0232 \mathrm{e}-01$ \\
\hline 9 & 1.4642 & $4.1314 \mathrm{e}-01$ & $9.2596 \mathrm{e}-03$ & $8.2924 \mathrm{e}-02$ & $1.7920 \mathrm{e}-02$ & $6.8817 \mathrm{e}-14$ & $1.3351 \mathrm{e}-12$ & $5.5650 \mathrm{e}-03$ & $1.0169 \mathrm{e}-01$ \\
\hline 10 & 1.4653 & $4.0919 \mathrm{e}-01$ & $9.4097 \mathrm{e}-03$ & $8.4462 \mathrm{e}-02$ & $1.8288 \mathrm{e}-02$ & $7.6530 \mathrm{e}-14$ & $1.5501 \mathrm{e}-12$ & $6.1564 \mathrm{e}-03$ & $1.1807 \mathrm{e}-01$ \\
\hline 11 & 1.4655 & $4.1277 \mathrm{e}-01$ & $9.4956 \mathrm{e}-03$ & $8.7030 \mathrm{e}-02$ & $1.7986 \mathrm{e}-02$ & $7.6449 \mathrm{e}-14$ & $1.5419 \mathrm{e}-12$ & $6.1474 \mathrm{e}-03$ & $1.1744 \mathrm{e}-01$ \\
\hline 12 & 5.5576 & $8.7013 \mathrm{e}-01$ & $2.7375 \mathrm{e}-03$ & $1.9644 \mathrm{e}-01$ & $2.4796 \mathrm{e}-02$ & 0.0000 & 0.0000 & 0.0000 & 0.0000 \\
\hline 13 & 5.6027 & $8.6371 \mathrm{e}-01$ & $2.4169 \mathrm{e}-03$ & $1.9313 \mathrm{e}-01$ & $2.5209 \mathrm{e}-02$ & 0.0000 & 0.0000 & 0.0000 & 0.0000 \\
\hline 14 & 1.4389 & $4.0085 \mathrm{e}-01$ & $1.0954 \mathrm{e}-02$ & $8.8157 \mathrm{e}-02$ & $1.6493 \mathrm{e}-02$ & $6.0265 \mathrm{e}-14$ & $1.1145 \mathrm{e}-12$ & $4.9122 \mathrm{e}-03$ & $8.4889 \mathrm{e}-02$ \\
\hline 15 & 1.4413 & $4.0665 \mathrm{e}-01$ & $1.1578 \mathrm{e}-02$ & $1.0250 \mathrm{e}-01$ & $1.6054 \mathrm{e}-02$ & $7.5335 \mathrm{e}-14$ & $1.5263 \mathrm{e}-12$ & $6.0593 \mathrm{e}-03$ & $1.1626 \mathrm{e}-01$ \\
\hline
\end{tabular}

it is not necessary to store the complete $A^{\lambda}$ and $B^{\lambda}$ matrices. If the number of non-zero elements are compared it is concluded that the memory consumption ${ }_{260}$ for $\alpha$-modes problem is higher than for the other kind of modes.

Table 5: Number of nonzero elements of matrices of $\lambda, \gamma$ and $\alpha$-modes problems.

\begin{tabular}{ccccc}
\hline Modes & ndofs & & \\
\hline & & $\operatorname{nnz}\left(A_{11}^{\lambda}\right)=\operatorname{nnz}\left(A_{22}^{\lambda}\right)$ & $\operatorname{nnz}\left(A_{21}^{\lambda}\right)$ & $\operatorname{nnz}\left(B_{11}^{\lambda}\right)=\operatorname{nnz}\left(B_{12}^{\lambda}\right)$ \\
$\lambda$-modes & $1.15 \mathrm{e}+5$ & $1.12 \mathrm{e}+7$ & $1.36 \mathrm{e}+7$ & $8.62 \mathrm{e}+6$ \\
\hline & & $\mathrm{nnz}\left(A^{\gamma}\right)$ & & $\mathrm{nnz}\left(B^{\gamma}\right)$ \\
$\gamma$-modes & $2.30 \mathrm{e}+5$ & $2.24 \mathrm{e}+7$ & $2.84 \mathrm{e}+7$ \\
\hline & & $\mathrm{nnz}\left(A^{\alpha}\right)$ & $\mathrm{nnz}\left(B^{\alpha}\right)$ \\
$\alpha$-modes & $2.30 \mathrm{e}+5$ & $4.22 \mathrm{e}+7$ & $2.24 \mathrm{e}+7$ \\
\hline
\end{tabular}

For solving the eigenproblems, first, Krylov-Schur method is applied for ordinary modes problems (27), (30) and (33) to obtain 4 eigenvalues, setting the Krylov subspace dimension to 21. The relative tolerance used is $\operatorname{res}_{o}=10^{-8}$ for $\lambda$ and $\gamma$-modes, and $\operatorname{res}_{o}=10^{-12}$ for $\alpha$-modes. This distinction is done to obtain good approximations (with residual errors less than $\operatorname{res}_{g}=10^{-5}$ ) in the generalized eigenvalue problem (see more details in Section 5.1). The KrylovSchur method has been initiated for all problems with an all-ones vector for the first eigenvector.

Table 5.2 displays the results for the first four eigenvalues together with the number of iterations of Krylov-Schur method, the mean number of iterations of BiCGStab, the residual error $\left(\operatorname{res}_{g}\right)$ and the CPU time necessary for the computations. All of these data are shown for the three configurations of reactor considered. It is observed that the reactor without perturbations is quasi-critical since the dominant $\lambda$ and $\gamma$ are near 1 , and $\alpha$ is near to 0 . In the Perturbation $275 \mathrm{I}$ and II, the reactor is subcritical with $k_{\text {eff }}=0.90$ and $k_{\text {eff }}=0.83$, respectively.

If the computational times are compared in the different configurations, it is observed that the computation for $\lambda$-modes is much quicker than the one for 
the other modes for similar residual errors. One reason is that for the $\lambda$-modes the problems have half size. The high times for the computation of $\gamma$-modes are due to the high number of iteration needed to converge the Krylov method since the spectrum of eigenvalues is clustered, as it has been already discussed for the homogeneous reactor. In the case of $\alpha$-modes, there are different reasons depending of the configuration of the reactor. For the critical configuration, the mean number of iterations of BiCGStab method used to solve the linear 285 systems is very high since the matrix $A^{\alpha}$ is ill-conditioned, but the number of iterations of Krylov method is low since the eigenvalues are relatively spaced. When the reactor is more subcritical the matrix $A^{\alpha}$ becomes better conditioned (since the eigenvalues are away from 0 ) and this is reflected in the number of iterations needed by the BiCGStab method. However the number of iterations of Krylov method is increased too because the eigenvalues are more clustered. Consequently, the computational times needed to compute the $\alpha$-modes are reduced as the reactor becomes more subcritical, but in any case the times remain larger than the times needed to compute the $\lambda$-modes.

Table 6: Eigenvalues in initial state of NEACRP reactor computed with Krylov-Schur method.

\begin{tabular}{|c|c|c|c|c|c|c|c|}
\hline \multirow[b]{2}{*}{ T. modes } & \multicolumn{3}{|c|}{ Eigenvalues } & \multirow[b]{2}{*}{$\begin{array}{l}\text { its. } \\
\text { Krylov }\end{array}$} & \multirow[b]{2}{*}{$\begin{array}{l}\text { mean its. } \\
\text { BiCGStab }\end{array}$} & \multirow[b]{2}{*}{ Res } & \multirow[b]{2}{*}{$\begin{array}{l}\text { CPU } \\
\text { Time (s) }\end{array}$} \\
\hline & $1 \mathrm{st}$ & 2nd & $3 \mathrm{th}=4 \mathrm{th}$ & & & & \\
\hline \multicolumn{8}{|c|}{ Critical State } \\
\hline$\lambda$-modes & 1.0002 & 0.9886 & 0.9854 & 13 & 14.9 & $1.5 \mathrm{e}-7$ & 105 \\
\hline$\gamma$-modes & 1.0001 & 0.9937 & 0.9919 & 22 & 10.6 & $6.1 \mathrm{e}-7$ & 661 \\
\hline$\alpha$-modes & 7.6341 & -442.32 & -573.62 & 3 & 109.7 & $2.2 \mathrm{e}-6$ & 735 \\
\hline \multicolumn{8}{|c|}{ Perturbation I } \\
\hline$\lambda$-modes & 0.0 .9093 & 0.8907 & 0.8958 & 13 & 14.9 & $1.5 \mathrm{e}-7$ & 106 \\
\hline$\gamma$-modes & 0.9490 & 0.9429 & 0.9412 & 23 & 10.6 & $5.0 \mathrm{e}-7$ & 669 \\
\hline$\alpha$-modes & -3462.14 & -3861.18 & -4006.92 & 4 & 31.9 & $3.7 \mathrm{e}-5$ & 438 \\
\hline \multicolumn{8}{|c|}{ Perturbation II } \\
\hline$\lambda$-modes & 0.8335 & 0.8238 & 0.8212 & 13 & 14.9 & $1.5 \mathrm{e}-7$ & 106 \\
\hline$\gamma$-modes & 0.9048 & 0.8990 & 0.8974 & 23 & 10.6 & $5.0 \mathrm{e}-7$ & 679 \\
\hline$\alpha$-modes & -6252.36 & -6610.89 & -6754.83 & 6 & 24.6 & $3.6 \mathrm{e}-5$ & 471 \\
\hline
\end{tabular}

The radial and axial profiles for the fast flux associated with the first three modes are shown in Figure 2 for critical configuration of the reactor. The radial profiles of fast flux functions are approximately equal for the first and second eigenvalues, observing small differences for the third one. In the last row of the figure, only the axial profiles associated with the first three $\lambda$-modes are shown because the axial profile obtained for the $\gamma$ and $\alpha$-modes are very close 300 to these ones. Furthermore, it is shown that the fast flux for the first modes is positive and has radial and axial symmetry, whereas the second modes are antisymmetric in axial profiles and symmetric in the radial ones. The third modes are antisymmetric in the radial and the axial profiles. 


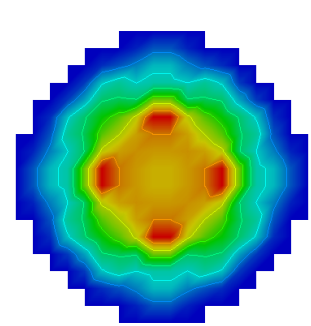

(a) Radial 1st $\lambda$-mode

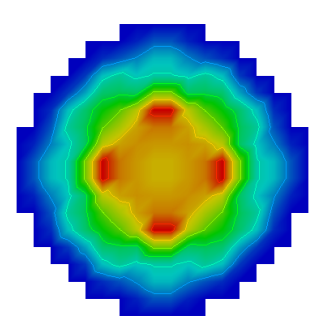

(d) Radial 1st $\gamma$-mode

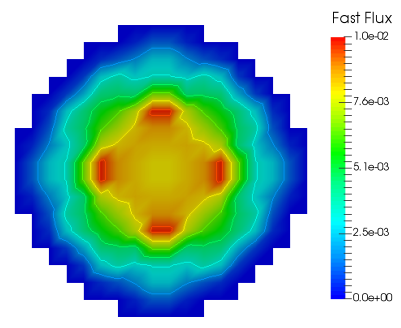

(g) Radial 1st $\alpha$-mode

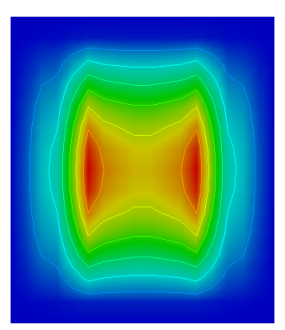

(j) Axial 1st $\lambda$-mode

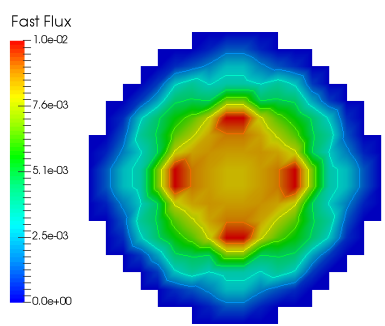

(b) Radial 2nd $\lambda$-mode

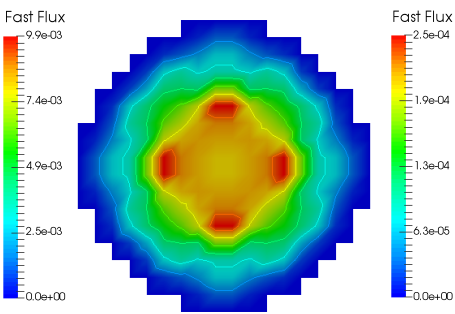

(e) Radial 2nd $\gamma$-mode

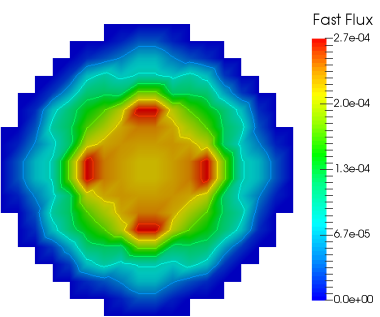

(h) Radial 2nd $\alpha$-mode

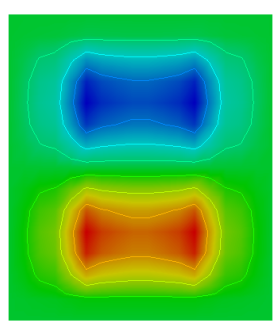

(k) Axial 2nd $\lambda$-mode

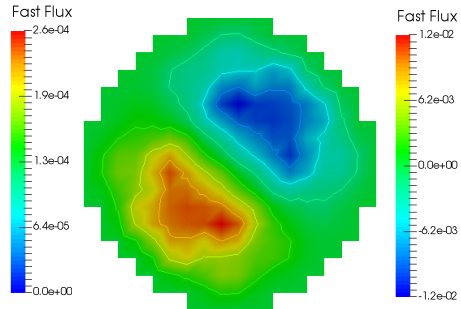

(c) Radial 3rd $\lambda$-mode

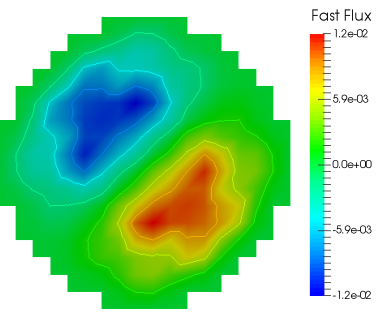

(f) Radial 3rd $\gamma$-mode

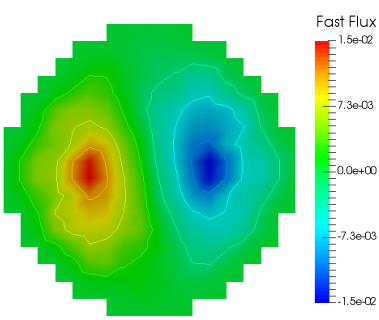

(i) Radial 3rd $\alpha$-mode

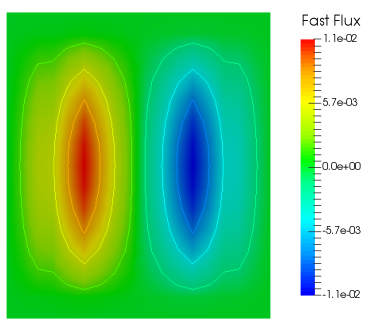

(l) Axial 3rd $\lambda$-mode

Figure 2: Radial and axial fast flux profiles for NEACRP in critical configuration.

The power average profiles (axial and radial) are used to compare the modes between them in the three configurations of the reactor. The axial profiles are 
computed as

$$
P_{a}(z)=\frac{\frac{1}{L_{x}} \frac{1}{L_{y}} \int_{0}^{L_{x}} \int_{0}^{L_{y}} \kappa_{1} \Sigma_{f 1}\left|\xi_{1}(\vec{r})\right|+\kappa_{2} \Sigma_{f 2}\left|\xi_{2}(\vec{r})\right| \mathrm{d} y \mathrm{~d} x}{\frac{1}{V_{t}} \int_{\Omega}\left(\kappa_{1} \Sigma_{f 1}\left|\xi_{1}\right|+\kappa_{2} \Sigma_{f 2}\left|\xi_{2}\right|\right) \mathrm{d} V},
$$

where $V_{t}, L_{x}$ and $L_{y}$ are the total volume, the width and the depth of the reactor core, respectively. The functions $\xi_{i}, i=1,2$ are the corresponding fast and thermal fluxes for the different kind of modes. The radial profiles are computed in a similar way. These profiles are shown in Figure 3. For critical configuration, there are no differences between the profiles of the modes. In the same way, there are no differences between the profiles of $\lambda$ and $\gamma$-modes in

310 Perturbation I and Perturbation II. However, when the reactor becomes more subcritical the $\alpha$-modes power shape changes with respect to its shape in the critical configuration and the other modes shapes in the radial profile.
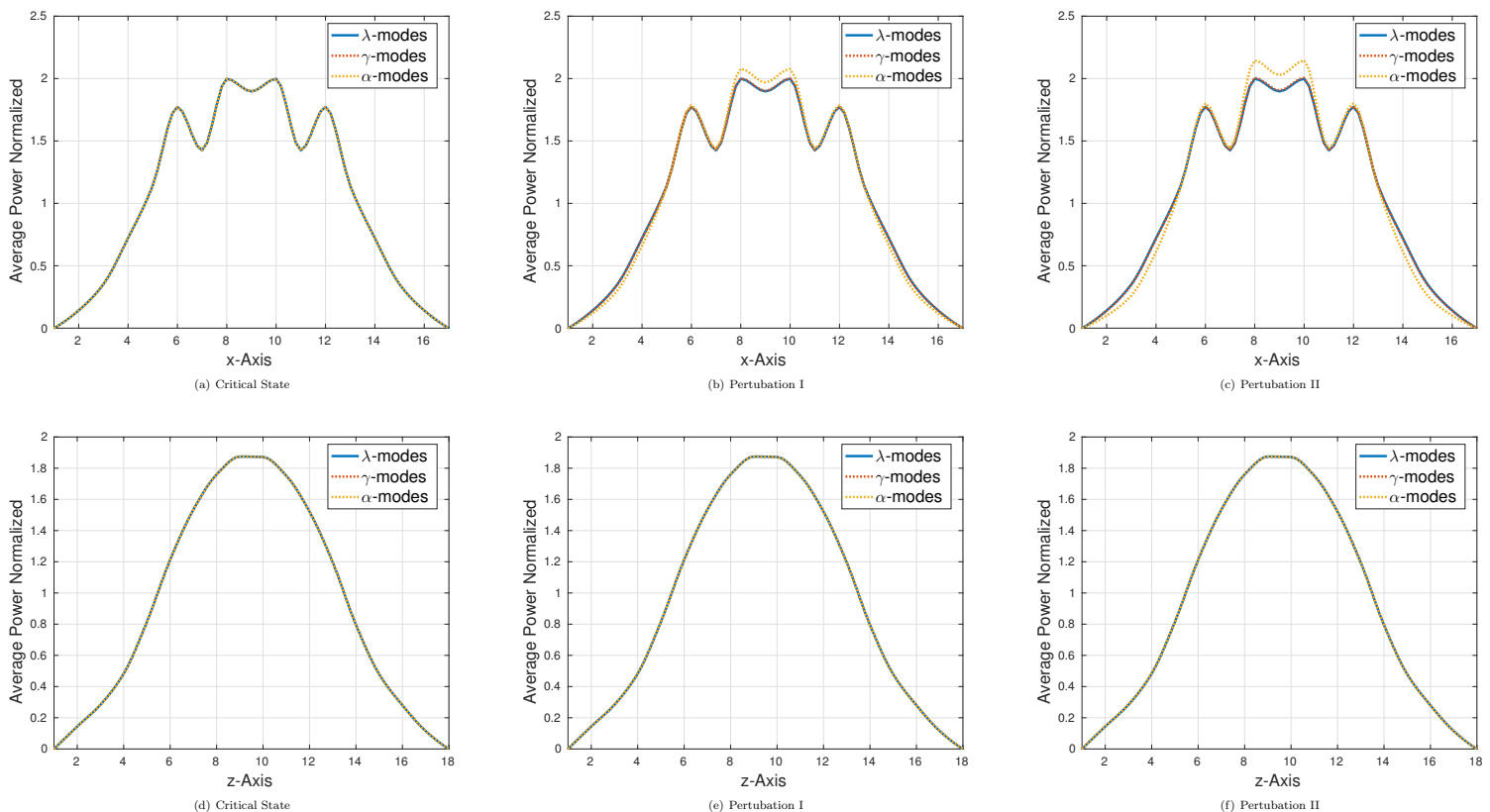

Figure 3: Average power profiles for the two configurations of NEACRP reactor.

As the computational time necessary to obtain the different modes with the Krylov-Schur method is very different (for $\alpha$ and $\gamma$-modes is much larger than 315 for $\lambda$-modes) and near of criticality the eigenfunctions are similar, it is proposed computing $\alpha$-modes and $\gamma$-modes using an alternative methodology that uses the $\lambda$-eigenvectors as an initial approximation. The proposed methods can be summarized as it is shown in Algorithm 2 and Algorithm 3. The stopping criterion used in these methods is based on the residual of the calculated eigenvalues and eigenvectors in generalized eigenvalue problem given by Eq. (60). 


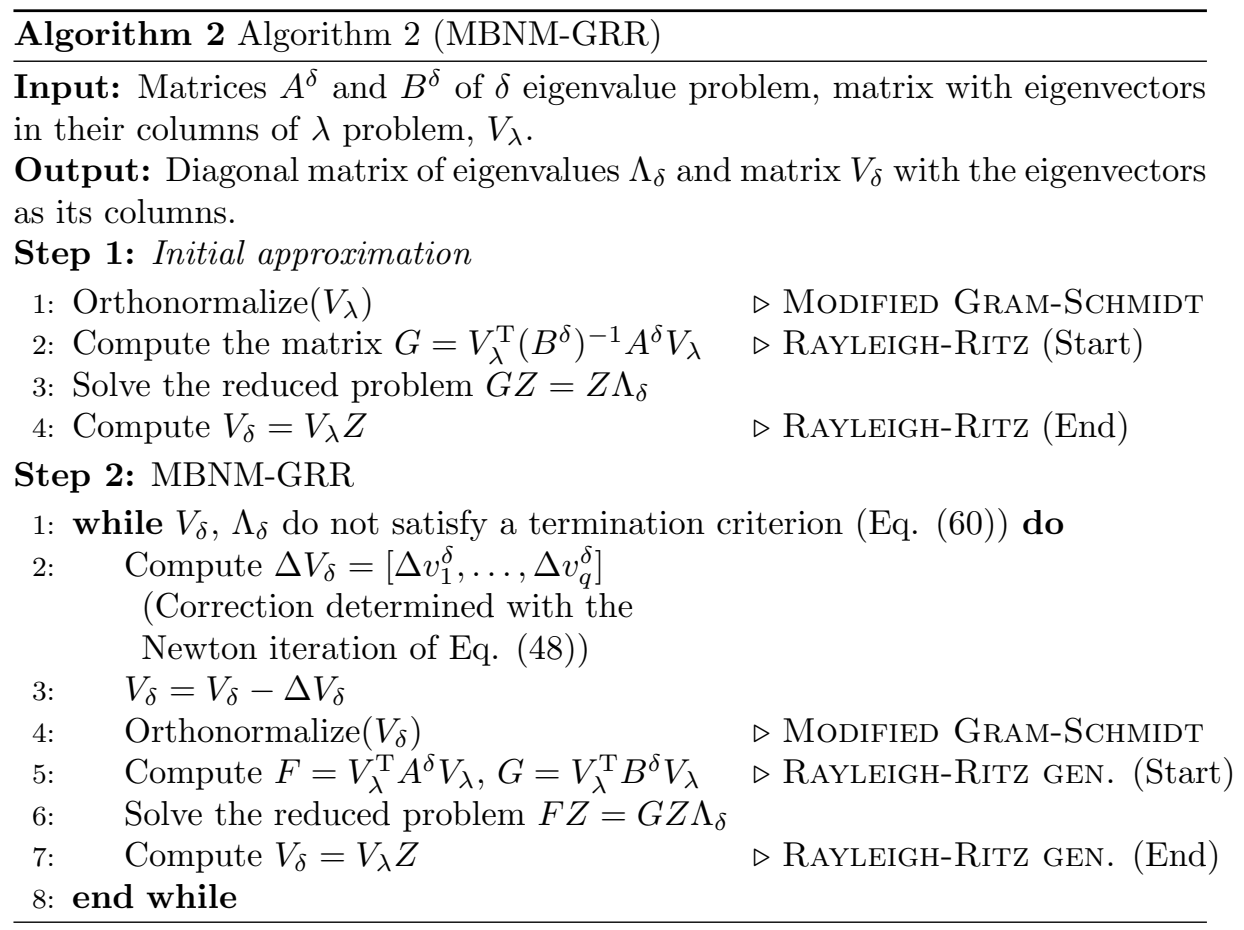

To compare the convergence of the different methods, many computations has been done using Krylov-Schur method modifying the required tolerance in the ordinary eigenvalue problem, computing the residual errors for the generalized eigenvalue problem (Eq. (60)) and obtaining their computational time necessary to converge the problem. For the block-Newton methods, we obtain, in each iteration, the computational time needed for the convergence and their residual error given by Eq (60). The time needed to compute the $\lambda$-modes to initiate both algorithms has been added in CPU time. Converged $\lambda$-modes have been used to initialize with a residual error $\operatorname{res}_{g}=10^{-7}$. Other strategies of 330 initialization based on considering different meshes are currently being studied (Carreño et al., 2017).

The convergence results are shown in Figure 4. For $\gamma$-modes, in critical configuration, the block-Newton methods are faster than the Krylov-Schur method. In the perturbed configurations, the initial errors obtained with the approximations of $\lambda$-modes are larger, but the block-Newton methods are more efficient than the Krylov method to obtain error lower than res $_{g}=10^{-3}$. With respect to the methods based on block-Newton method, the convergence of MBNM-GRR is slightly faster than the one of MBNM-GBO method.

For the $\alpha$-modes there are more differences. The results for MBNM-GBO are not included since this method does not converge for these modes. For the critical configuration, the MBNM-GRR is more efficient than the KrylovSchur method, in fact there is a difference of 200 seconds between both methods 


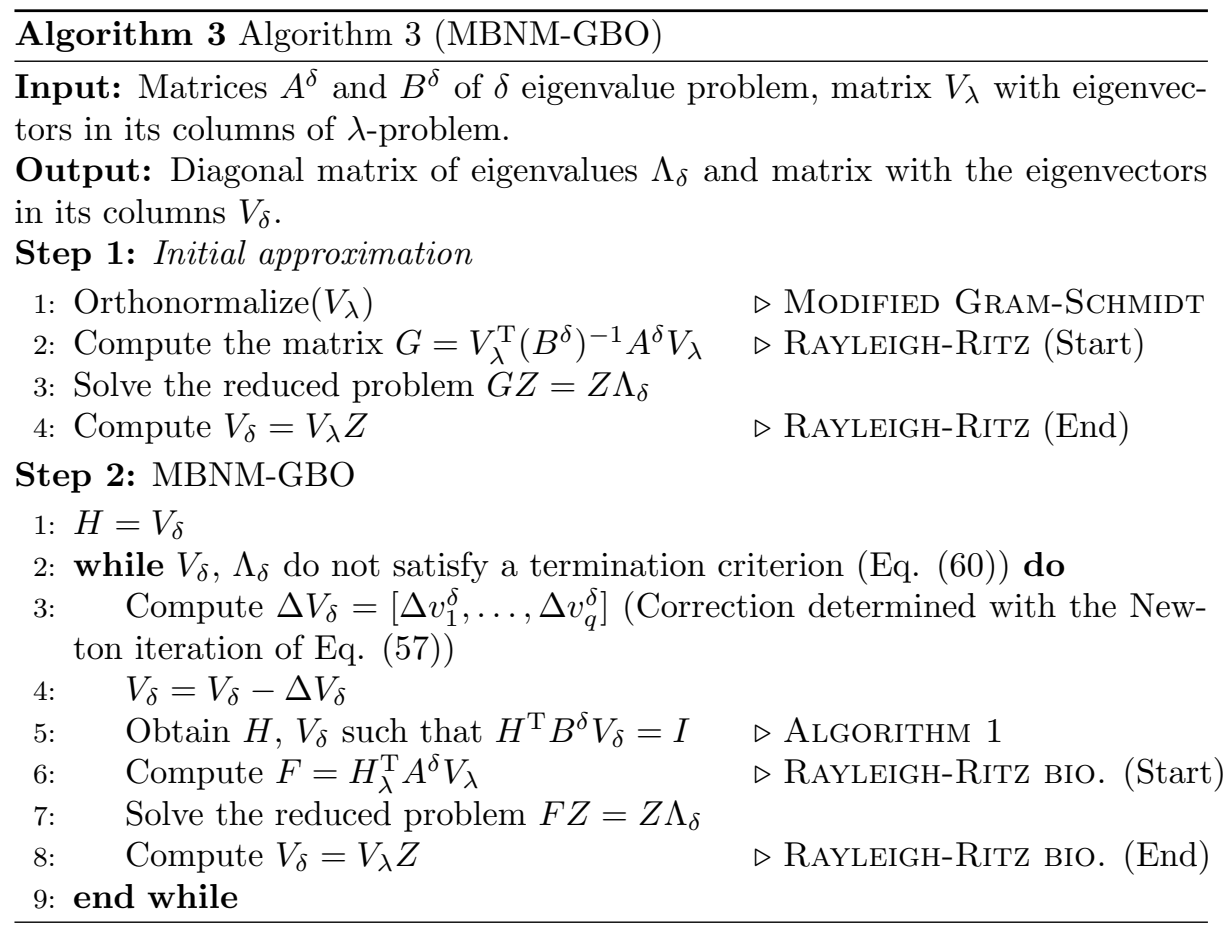

to obtain a residual error of $\operatorname{res}_{g}=10^{-6}$. In Perturbation I, the speed of the method is similar, however for residual errors lower than $\operatorname{res}_{g}=10^{-1}$ the 345 MBNM-GRR computes the solution in less time than Krylov-Schur method. In Perturbation II the shape of $\alpha$-modes is more different from the shape of the $\lambda$-modes and this makes that the MBNM-GRR method is not as fast as Krylov-Schur method.

\subsubsection{Spectral index}

To demonstrate the variation in the neutron energy spectrum, the spectral index $I$ is studied. This index is defined as the ratio of the fluxes integrated in the core volume (Ronen et al., 1976),

$$
I=\frac{\int_{\Omega} \xi_{1} \mathrm{~d} V}{\int_{\Omega} \xi_{2} \mathrm{~d} V}
$$

where $\xi_{1}, \xi_{2}$, are the fast and thermal fluxes of $\lambda, \alpha$ and $\gamma$-modes.

16 configurations of NEACRP benchmark have been considered to study the spectral index behavior of the $\lambda, \gamma$ and $\alpha$-modes. These configurations are obtained modifying the position of central control rod, since in the case A1 of NEACRP benchmark, the central control rod is moved. In Figure 5, the differences between the spectral indexes $(I)$ and the spectral index when the reactor is in critical configuration $\left(I_{c}\right)$ are represented in each configuration as 
a function of the $\lambda$-eigenvalue. In Figure 5(a), we observe that near criticality these differences in each mode are nearly equal and increasing, but from $\lambda=$ 1.001 these functions are separated and for $\lambda=1.003$ they become decreasing functions. Also, in Figure 5(b), we observe that functions are similar when the first $\lambda$-eigenvalue is close to 1 . For the second mode, a relative maximum is observed for $\lambda_{2}=1$. So, the spectral indexes for $\lambda, \gamma$ and $\alpha$-modes have the same behavior.

\section{Conclusions}

365

\section{Acknowledgements}

This work has been partially supported by Spanish Ministerio de Economía

395 y Competitividad under projects ENE2014-59442-P, MTM2014-58159-P and BES-2015-072901.

We authors also want to thank the anonymous reviewers for their comments and suggestions that have greatly improved the manuscript. 


\section{References}

400 Adrover, A., Creta, F., Giona, M., \& Valorani, M. (2005). Biorthogonalization, geometric invariant properties and rate-based estimate of Lyapunov spectra. Physics Letters A, 342, 421-429.

Avvakumov, A. V., Strizhov, V. F., Vabishchevich, P. N., \& Vasilev, A. O. (2017). Spectral properties of dynamic processes in a nuclear reactor. Annals of Nuclear Energy, 99, 68-79.

Bangerth, W., Hartmann, R., \& Kanschat, G. (2007). deal.II - a general purpose object oriented finite element library. ACM Trans. Math. Softw., 33, 24/1$24 / 27$.

Bell, G. I., \& Glasstone, S. (1970). Nuclear Reactor Theory. New York: Litton Educational Publishing, INC.

Carreño, A., Vidal-Ferrandiz, A., Ginestar, D., \& Verdú, G. (2017). Multilevel method to compute the lambda modes of the neutron diffusion equation. Applied Mathematics and Nonlinear Sciences, 2, 225-236.

Finnemann, H. (). A consistent nodal method for the analysis of space-time effects in large lwr's.

Finnemann, H., \& Galati, A. (1991). NEACRP 3-D LWR core transient benchmark, final specification. NEACRPL-335 (Revision 1), .

González-Pintor, S., Ginestar, D., \& Verdú, G. (2011). Updating the lambda modes of a nuclear power reactor. Mathematical and Computer Modelling, 54, 1796-1801.

Gupta, A., \& Modak, R. (2011). Evaluation of dominant time-eigenvalues of neutron transport equation by meyers sub-space iterations. Annals of Nuclear Energy, 38, 1680-1686.

Hébert, A. (1987). Development of the nodal collocation method for solving the neutron diffusion equation. Annals of Nuclear Energy, 14, 527-541.

Henry, A. F. (1975). Nuclear-reactor analysis volume 4. MIT press Cambridge, Massachusetts.

Hernandez, V., Roman, J. E., \& Vidal, V. (2005). SLEPc: A scalable and flexible toolkit for the solution of eigenvalue problems. ACM Trans. Math. Software, 31, 351-362.

Kópházi, J., \& Lathouwers, D. (2012). Three-dimensional transport calculation of multiple alpha modes in subcritical systems. Annals of Nuclear Energy, 50, 167-174.

Lathouwers, D. (2003). Iterative computation of time-eigenvalues of the neutron transport equation. Annals of Nuclear Energy, 30, 1793-1806. 
Lewins, J. (2013). Nuclear reactor kinetics and control. Elsevier.

Li, R.-C. (2014). Rayleigh quotient based optimization methods for eigenvalue problems. Matrix Functions and Matrix Equations, 19, 76-108.

Lösche, R., Schwetlick, H., \& Timmermann, G. (1998). A modified block Newton iteration for approximating an invariant subspace of a symmetric matrix. Linear Algebra and its Applications, 275, 381 - 400.

March-Leuba, J., \& Blakeman, E. (1991). A mechanism for out-of-phase power instabilities in boiling water reactors. Nuclear Science and Engineering, 107, $173-179$.

Miró, R., Ginestar, D., Hennig, D., \& Verdu, G. (2000). On the regional oscillation phenomenon in BWR's. Progress in Nuclear Energy, 36, 189-229.

Miró, R., Ginestar, D., Verdú, G., \& Hennig, D. (2002). A nodal modal method for the neutron diffusion equation. Application to BWR instabilities analysis. Annals of Nuclear Energy, 29, 1171-1194.

Modak, R., \& Gupta, A. (2007). A scheme for the evaluation of dominant timeeigenvalues of a nuclear reactor. Annals of Nuclear Energy, 34, 213-221.

Ronen, Y., Shvarts, D., \& Wagschal, J. (1976). A comparison of some eigenvalues in reactor theory. Nuclear Science and Engineering, 60, 97-101.

Saad, Y. (1992). Numerical methods for large eigenvalue problems. SIAM.

Saad, Y. (2003). Iterative methods for sparse linear systems. SIAM.

Singh, K., Degweker, S., Modak, R., \& Singh, K. (2011). Iterative method for obtaining the prompt and delayed alpha-modes of the diffusion equation. Annals of Nuclear Energy, 38, 1996-2004.

Singh, T., Mazumdar, T., \& Pandey, P. (2014). NEMSQR: A 3-D multi group diffusion theory code based on nodal expansion method for square geometry. Annals of Nuclear Energy, 64, 230 - 243.

Smith, K. (1979). An analytic nodal method for solving the two-group, multidimensional, static and transient neutron diffusion equations. Ph.D. thesis Dept. of Nuclear Engineering, Massachusetts Institute of Technology.

Stacey, W. M. (2007). Nuclear reactor physics. John Wiley \& Sons.

Stewart, G. W. (2002). A Krylov-Schur algorithm for large eigenproblems. SIAM Journal on Matrix Analysis and Applications, 23, 601-614.

Uyttenhove, W., Lathouwers, D., Kloosterman, J.-L., van der Hagen, T., Van den Eynde, G., \& Baeten, P. (2014). Methodology for modal analysis at pulsed neutron source experiments in accelerator-driven systems. Annals of Nuclear Energy, 72, 286-297. 
Velarde, G., Ahnert, C., \& Aragones, J. (1978). Analysis of the eigenvalue equations in k, lambda,. gamma. , and. cap alpha. applied to some fast- and thermal-neutron systems. Nucl. Sci. Eng., 66:3.

475 Verdú, G., Ginestar, D., Miró, R., \& Vidal, V. (2005). Using the JacobiDavidson method to obtain the dominant lambda modes of a nuclear power reactor. Annals of nuclear energy, 32, 1274-1296.

Verdu, G., Ginestar, D., Roman, J., \& Vidal, V. (2010). 3D alpha modes of a nuclear power reactor. Journal of nuclear science and technology, 47, 501514 .

Verdú, G., Ginestar, D., Vidal, V., \& Miró, R. (1998). Modal decomposition method for BWR stability analysis. Journal of nuclear science and technology, $35,538-546$.

Verdú, G., Ginestar, D., Vidal, V., \& Muñoz-Cobo, J. (1994). 3D $\lambda$-modes of the neutron-diffusion equation. Annals of Nuclear Energy, 21, 405-421.

Verdú, G., Miró, R., Ginestar, D., \& Vidal, V. (1999). The implicit restarted Arnoldi method, an efficient alternative to solve the neutron diffusion equation. Annals of nuclear energy, 26, 579-593.

Vidal-Ferrandiz, A., Fayez, R., Ginestar, D., \& Verdú, G. (2014). Solution of the lambda modes problem of a nuclear power reactor using an $h-p$ finite element method. Annals of Nuclear Energy, 72, 338 - 349.

Wang, Y., Bangerth, W., \& Ragusa, J. (2009). Three-dimensional h-adaptivity for the multigroup neutron diffusion equations. Progress in Nuclear Energy, $51,543-555$. 


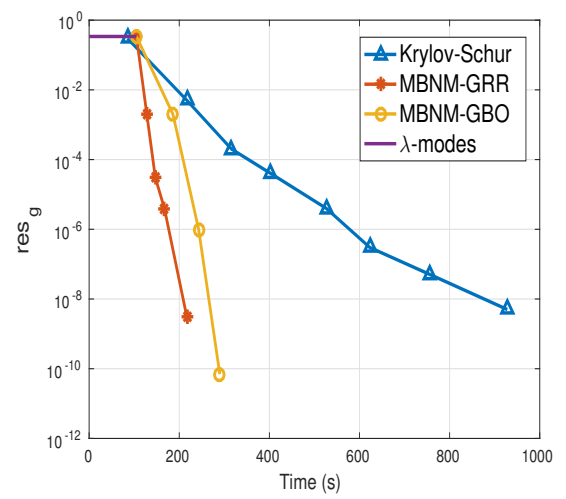

(a) $\gamma$-modes: critical configuration

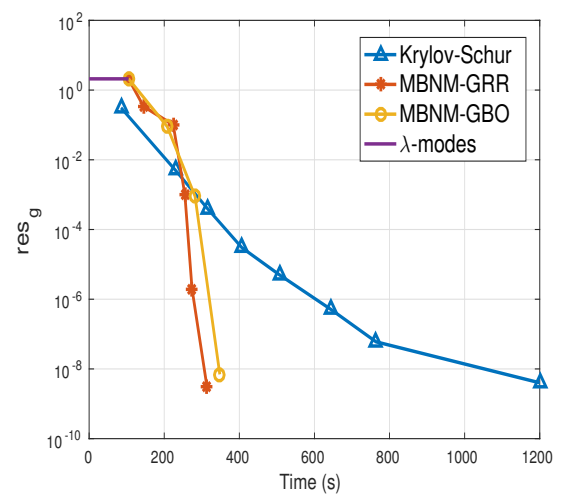

(c) $\gamma$-modes: Perturbation I

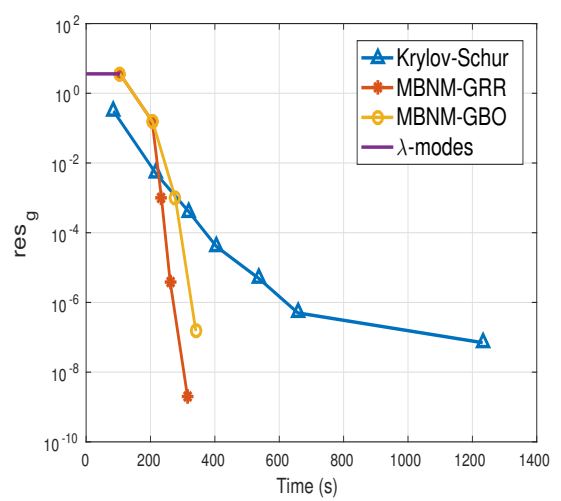

(e) $\gamma$-modes: Perturbation II

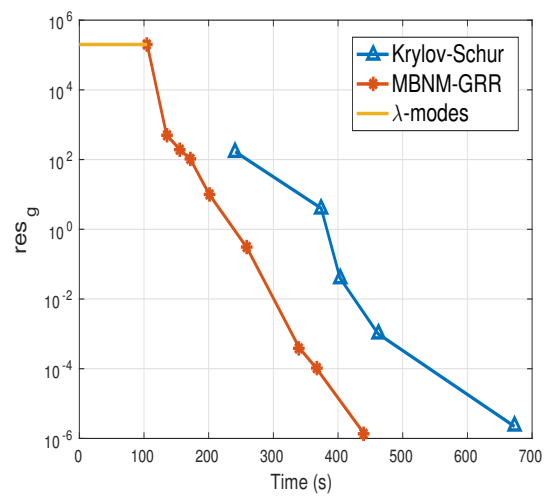

(b) $\alpha$-modes: critical configuration

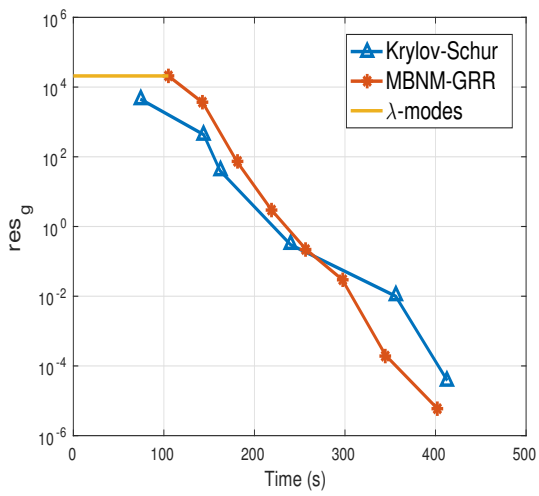

(d) $\alpha$-modes: Perturbation I

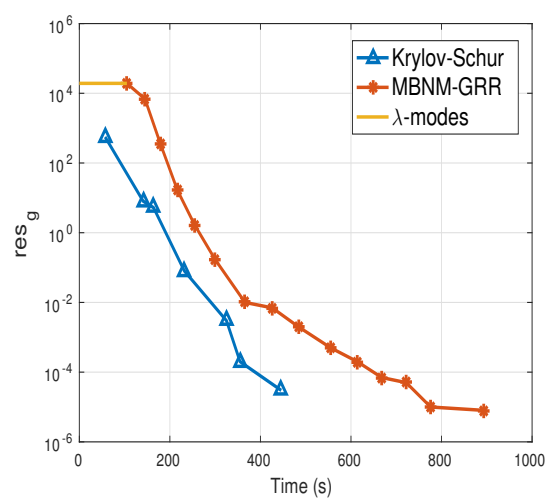

(f) $\alpha$-modes: Perturbation II

Figure 4: Residual error against CPU time for the NEACRP reactor with Krylov-Schur, MBNM-GRR and MBNM-GBO methods. 


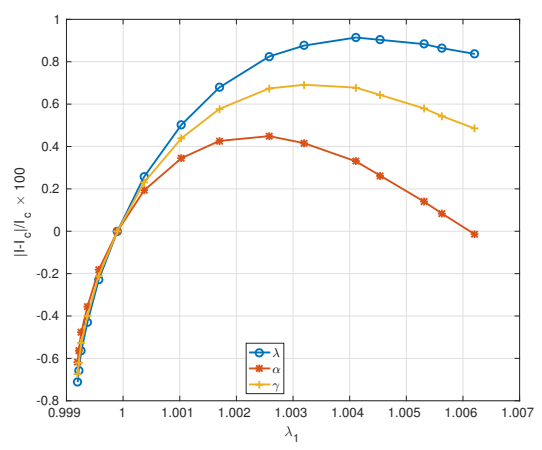

(a) First mode

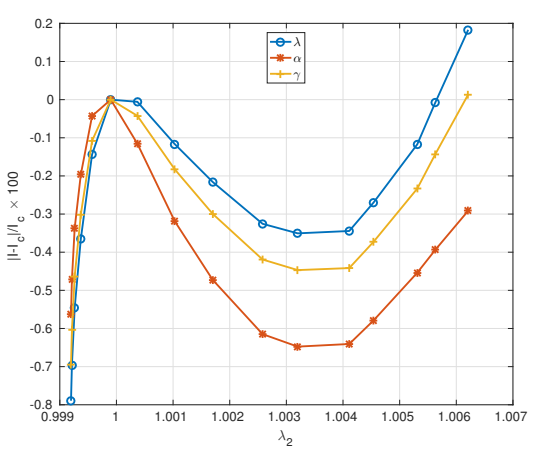

(b) Second mode

Figure 5: Spectral indexes in NEACRP reactor. 